Circulation et archipels de l'exception

\title{
Histoires peintes. Des artistes au camp de Beddawi
}

Painted stories. Artists in the Beddawi camp

Amanda S. A. Dias

\section{(2) OpenEdition}

\section{Journals}

\section{Édition électronique}

URL : http://journals.openedition.org/conflits/6953

DOI : 10.4000/conflits.6953

ISSN : $1777-5345$

Éditeur :

CCLS - Centre d'études sur les conflits lilberté et sécurité, L'Harmattan

\section{Édition imprimée}

Date de publication : 30 décembre 2007

Pagination : 149-164

ISBN : 978-2-296-05120-1

ISSN : 1157-996X

Référence électronique

Amanda S. A. Dias, « Histoires peintes. Des artistes au camp de Beddawi », Cultures \& Conflits [En ligne], 68 | hiver 2007, mis en ligne le 18 avril 2008, consulté le 30 mars 2021. URL : http:// journals.openedition.org/conflits/6953; DOI : https://doi.org/10.4000/conflits.6953 


\title{
Histoires peintes. \\ Des artistes au camp de Beddawi
}

\author{
Amanda S. A. DIAS
}

Amanda S. A. Dias est doctorante en co-tutelle internationale entre l'EHESS et l'université de l'Etat de Rio de Janeiro (UERJ).

«La peinture, même le dessin, c'est un art qui aime expliquer les problèmes aux peuples; sinon ce n'est pas de l'art. Il faut chercher les manières de résoudre ce problème. Les difficultés pour vivre, la position économique, la pauvreté... Comment peut-on l'expliquer ? Par exemple, l'auteur écrit : c'est un art, c'est l'art d'écrire; le poète fait un poème : c'est un art; les gens du théâtre, les artistes du théâtre font des pièces pour résoudre un certain problème. Donc les peintres, ils doivent s'interroger sur les problèmes qu'ils rencontrent. Pour moi, quel est le problème le plus important ? C'est le problème de la Palestine. Je parle du problème palestinien. Mais à l'aide des personnes. Et tu vois que tous les tableaux parlent, ou bien cherchent à résoudre, ou à faire comprendre, le problème palestinien. [...] Comment me demander d'oublier le problème principal de ma vie? Je pense que tu as vu que je pleure lorsque je parle de comment j'ai quitté la Palestine. Comment me demander de quitter et d'oublier mon pays ? ${ }^{1}$ »

T e camp de Beddawi se situe dans les collines à $5 \mathrm{~km}$ au nord de Tripoli (Liban). Etabli par l'UNRWA 2 en 1955, il est, avec Nahr Al-Bared, l'un des deux camps de réfugiés reconnus par l'agence onusienne au Nord-Liban. Entre autres particularités, ce camp rassemble une petite communauté d'artistes plasticiens. Ils sont pour la plupart palestiniens et ils sont réfugiés.

1. Propos de monsieur Buhrân, avril 2004.

2. Office de secours et de travaux des Nations unies pour les réfugiés de Palestine dans le ProcheOrient. 
Le présent dossier prend appui sur un travail de terrain réalisé en plusieurs étapes dans le camp de Beddawi au Nord Liban ${ }^{3}$. Parallèlement à la présentation de certaines œuvres ${ }^{4}$, nous avons voulu faire entendre la voix de ces peintres, en reprenant des fragments d'entretiens réalisés en 2004, où l'« exceptionnel » de leur situation est sans cesse rappelé comme « cause à défendre », motif inéluctable de ce que l'on peint, et rendre compte du plus quotidien, du plus routinier, de la vie de ces peintres et de leur entourage, au sein de ce camp. Pour ce faire, outre les entretiens, nous mobilisons le carnet de route établi au cours de l'un de nos voyages, pour raconter le déroulement d'une journée à Beddawi. Le dossier ainsi constitué donne à entendre plusieurs voix et chacune d'entre elles raconte une histoire, à sa manière et dans sa langue. La langue dans laquelle chacun s'exprime n'est pas un détail de cette enquête. Certains entretiens ont été réalisés en français, d'autres en anglais et en arabe (nous avons alors eu recours à une traduction simultanée) ${ }^{5}$. Ainsi, dans certains cas, plusieurs traductions ont été nécessaires pour parvenir à la version que nous présentons ici. Nous avons choisi autant que possible de respecter la langue des peintres rencontrés et de ne pas procéder à des «corrections ». Nous comprenons que «les mots pour le dire » ont un sens, une raison d'être, et que c'est à nous, auditeurs, lecteurs, de tendre l'oreille pour mieux entendre.

\section{Antécédents}

L'histoire des réfugiés palestiniens remonte à 1947, date du vote du partage de la Palestine en deux Etats ${ }^{6}$. Fuyant leurs maisons et leurs terres en quatre vagues successives, entre 1947 et 1949, les Palestiniens sont partis en grand nombre au Liban et dans les pays voisins. Avec une population de plus de quatre millions, ils représentent aujourd'hui le plus grand groupe de réfugiés dans le monde.

Au Liban, la quasi-totalité des Palestiniens est originaire de Galilée et des villes côtières. En 1948, environ 100000 à 130000 réfugiés sont arrivés dans le pays des Cèdres. Pendant les deux premières années, la Ligue des sociétés de la Croix-Rouge était la principale organisation portant assistance aux réfugiés

3. Peintres de Beddawi. Création artistique et imaginaire politique dans un camps de réfugiés palestiniens au Liban, mémoire de DEA en sociologie, EHESS, dir. H. Bozarslan, septembre 2004. Ce mémoire est disponible dans la collection «Masters» du réseau scientifique TERRA : http://terra.rezo.net. Voir également l'article « Peintres de Beddawi. Entre création artistique et engagement politique », à paraittre dans Mermier F., Puig N. (dirs.), Itinéraires esthétiques et scènes culturelles au Proche-Orient, Beyrouth, éditions IFPO, 2007.

4. Voir site Internet de la revue : www.conflits.org

5. Nizar, l'un des artistes du camp, a fait la traduction simultanée des entretiens de l'arabe vers l'anglais. Nizar n'a jamais demandé d'argent pour ce service. Ce travail sur les artistes du camp était important pour lui et il a fait tout ce qu'il pouvait pour le rendre plus facile.

6. Le 29 novembre 1947, la résolution 181 des Nations unies adopte un plan de partage de la Palestine. Il prévoit que, le $1^{\mathrm{er}}$ août 1948 , lorsque le mandat britannique aura pris fin, le pays sera partagé en deux Etats indépendants, l'un arabe, l'autre juif. 
palestiniens. En 1950, l'UNRWA prend le relais. Cette agence de l'ONU chargée du secours des réfugiés, assure des services sociaux, ainsi que des services en matière d'éducation, de santé et des aides d'urgence aux Palestiniens vivant dans la bande de Gaza, en Cisjordanie, en Jordanie, au Liban et en Syrie.

Actuellement, près de 406000 réfugiés palestiniens sont inscrits auprès de l'UNRWA au Liban. A ce nombre, il faudrait ajouter entre 15000 et 16000 réfugiés de la guerre israélo-arabe de 1967. Plus de la moitié de ces réfugiés au Liban (53\%) habite dans les 12 camps officiels établis par l'Agence onusienne. Le Liban est ainsi le pays qui présente aujourd'hui la proportion la plus importante de Palestiniens habitant encore dans ces espaces en marge ; un signe de leur non-intégration au sein de la société libanaise. D'après l'UNRWA, ces camps témoignent de la précarité de la condition des réfugiés au Liban : absence d'infrastructures appropriées, surpeuplement, pauvreté, chômage, etc.

De plus, le système politique libanais basé sur la répartition confessionnelle du pouvoir constitue un problème fondamental dans la gestion des affaires des réfugiés. L'intégration des Palestiniens a toujours représenté une menace pour le fragile équilibre confessionnel du pays. La législation libanaise régissant les droits des réfugiés palestiniens limite l'accès des réfugiés au monde du travail, à l'éducation, aux services sociaux, à la propriété et à la mobilité internationale.

En ce qui concerne l'emploi, soixante-douze métiers leur sont interdits. En juin 2005, le ministre libanais du Travail a promulgué le « Mémorandum n67/1 », autorisant les réfugiés palestiniens à obtenir des permis de travail, difficiles à obtenir auparavant. Cependant, le mémorandum ne concerne pas les diplômés palestiniens, qui ne peuvent toujours pas exercer la médecine, le droit, l'architecture... Ainsi, l'impact du mémorandum n'est pas, ou peu, perceptible. Il n'y a pas de statistiques officielles, mais l'UNRWA estime que $60 \%$ des réfugiés palestiniens vivent en dessous du seuil de pauvreté et que leur taux de chômage atteindrait $70 \%$.

Par ailleurs, la loi no291 d'avril 2001 interdit à «quiconque n'a pas de nationalité dans un Etat reconnu » d'être propriétaire. Cela prive donc les Palestiniens du droit d'acheter des maisons et des biens immobiliers en général au Liban. Elle les prive aussi du droit à l'héritage de la propriété. Les réfugiés palestiniens sont également soumis à des restrictions concernant l'entrée de matériaux de construction dans les camps de réfugiés, ce qui empêche tout entretien ou rénovation des logements. En 2005, ces restrictions ont été levées, mais elles pourraient à nouveau être imposées sur simple décision des autorités libanaises.

\section{Le camp de Beddawi}

D'après l'UNRWA, Beddawi comptait 15947 réfugiés inscrits d'origine palestinienne en décembre 2006. Vivent également dans le camp environ 
400 personnes d'origine palestinienne non inscrites, et entre 1400 et 1500 personnes d'autres nationalités (Libanais, Syriens, Kurdes...). Issus de milieux sociaux très modestes, les non-Palestiniens de Beddawi sont attirés par les loyers à bas prix des logements. D’une superficie inférieure à deux kilomètres carrés, le camp, déjà surpeuplé, a vu sa population doubler à la suite des affrontements qui ont eu lieu du 20 mai au 2 septembre 2007 entre l'armée libanaise et les militants du groupe islamiste Fatah Al-Islam. Les deux principaux problèmes du camp de Beddawi sont la pauvreté et le chômage. En décembre 2003, 644 familles, soit 2993 personnes, étaient inscrites comme «Special Hardship Cases » auprès du programme d'aide sociale de l'Agence soit $20 \%$ de la population. Ce programme offre une assistance matérielle et financière directe aux réfugiés considérés comme les plus vulnérables : veuves, femmes divorcées ou abandonnées, personnes âgées, réfugiés handicapés ou très jeunes. 254 patients par jour sont soignés au dispensaire de l'UNRWA. 3268 élèves sont inscrits en 2006-2007 dans les six écoles de l'UNRWA élémentaires/préparatoires fonctionnant en double cursus ${ }^{7}$ et dans l'école secondaire, ainsi qu'une cinquantaine d'enfants dans l'école maternelle francophone.

Un certain nombre d'ONG sont actives à Beddawi, dont Al-Najda, Beit Atfal Al-Soumoud, Ghassan Kanafani Cultural Foundation, Lina Nabulsi National Association et la Palestinian Red Cross Society ainsi que de nombreuses ONG internationales comme Handicap international, Première Urgence, Save the Children...

\section{Des voix à Beddawi}

A Beddawi, les artistes se connaissent entre eux. Ils entretiennent des relations professionnelles et amicales. Comme la quasi-totalité des Palestiniens au Liban, ils sont tantôt issus de la génération des réfugiés de 1948 tantôt parents de cette génération, originaires de Galilée et des villes côtières du Nord de la Palestine. Parmi eux, un seul, monsieur Burhân, appartient à la première génération arrivée au Liban. Ancien professeur de l'UNRWA, âgé de 66 ans, monsieur Burhân est un artiste autodidacte qui, depuis sa retraite, a la possibilité de se consacrer exclusivement à son travail artistique. Il est arrivé au camp de Beddawi en 1958 :

"Je suis dans un village appelé Zar Natar, j'ai sept ans, presque sept ans, le bombardement du village commence par les avions israéliens, ceux-là sont britanniques, mais ce sont les mêmes... On n'a pas d'armes [...] il n'y a pas d'armée palestinienne, il n'y a pas d'armes chez les gens du village. C'est un village pauvre qui vit en cultivant la terre... Les gens du village commencent à fuir. Moi et

7. «Double shift» en anglais. Comme il n'y a pas assez d'espace dans le camp, et que le nombre d'élèves y est très élevé, deux écoles sont obligées de partager un même espace. Une école fonctionne le matin, l'autre l'après-midi. 
ma famille, nous restons. L'armée juive entre dans le village et ils réunissent les gens sur une place, dans le village il y a une grande place [...]. L’après-midi ils nous disent : “allez-vous en, allez dans les terres arabes”; et ils commencent à mitrailler, avec les mitrailleurs ils commencent à tirer en l'air. Sur la route, il y a un pont qu'il faut traverser, c'est la route qui relie les villages. Arrivant à mi-chemin sur le pont, un groupe de tanks descend si rapidement et tire avec les mitrailleurs. Les gens se mettent à fuir et ils se dispersent. Quelqu'un monte la colline, quelqu'un qui descend la vallée, quelqu'un qui rentre dans les arbres des olives... j'ai une petite sœur, elle doit avoir un an. Je descends avec ma petite sœur, après une heure, on commence à chercher nos parents [...]. Un des mes voisins me trouve, il me demande : Où sont vos parents ?" Je lui dis : "Je ne sais pas". Il me prend avec lui et me met sur la route. Sur la route on a trouvé mon père et ma mère, ensuite mes frères. Je porte ma petite sœur sur les épaules et on commence à traverser les montagnes situées entre la Palestine et le Liban.

Arrivés au Liban, nous nous installons une nuit dans un village appelé El Mash. Le deuxième jour nous allons à Beteshbel [...] la Croix-Rouge donne quelques aides pour les réfugiés au Liban pour presque une semaine. Un train vient et nous apporte jusqu'ici à Tripoli, au Mina, jusqu'à la gare [...]. Pour les premiers quinze jours nous nous installons dans les wagons du train. Ce sont des wagons pour transporter les animaux, comme les moutons, les chèvres, les bœufs, etc., entre les pays arabes et la Turquie. Après quinze jours, la pluie commence à tomber. Les fissures trouvées sur le toit du wagon commencent à... Nous commençons à quitter le wagon, nous descendons des wagons, nous sommes allés dans les logements. Chaque logement comporte sept familles, quinze familles, etc. Pendant quelques mois nous commençons à connaître l'entourage, je commence à ramasser dans les rues des cigarettes jetées. Les cigarettes jetées on fait comme ça, on les déchire, on fait sortir le tabac comme ça, on les réunit et lorsqu'on obtient une grande quantité on commence à les vendre pour les gens qui fument. Une fois quand je ramassais les cigarettes par terre un homme qui rasait les barbes m'appelle et me demande : “- Qu'est-ce que tu fais ?” Je lui dis : “- Je ramasse les cigarettes jetées par terre. - Pourquoi ? Pour les vendre aux gens”. Il me fait demander à mon père si je peux travailler chez lui. Mon père accepte parce qu'on n'a pas d'argent et il faut l'avoir pour pouvoir vivre. Je commence à travailler chez le barbier [...] Le barbier me prend à l'école, m'enregistre et il me demande de revenir après l'école [...] Je commence à étudier et à travailler en même temps. Nous avons loué une petite chambre dans un endroit à Mina [...]. 
Il faut aller du travail à l'école en traversant dix kilomètres. Ici, à droite il y a le cimetière des chrétiens. A gauche, il y a le cimetière des musulmans. Lorsque j'arrive au bout de la route, je suis debout et je me demande qu'est-ce que je fais. Je prends le sac sur mon dos et je commence à courir. Chaque jour il faut courir [...] un kilomètre et demi. Après le cimetière, il y a une route, c'est affreux pour un enfant [...] je suis obligé de traverser la route, je commence à courir. Une fois un homme dans le cimetière chrétien me voit courir. Le deuxième jour je l'ai trouvé debout dans le même lieu où je me suis mis à penser qu'est-ce qu'il faut faire. Il me dit : "qu'est-ce que tu as ?" Je lui dit que j'ai peur. Il prend ma main et me dit de venir. Il me fait arriver à la fin du chemin. Chaque jour, il m'emmène pendant presque qu'une année.

A l'époque je travaille après l'école, je fais comme ça jusqu'au brevet. Après le brevet, j'ai étudié le baccalauréat dans une autre école et j'ai quitté le travail. Après avoir terminé le baccalauréat je suis parti dans une école spéciale. Le directeur me demande si je veux apprendre à dessiner dans l'école [...] je commence à apprendre le dessin dans cette école et après quelques années je l'ai quittée pour entrer à l'UNRWA, parce que l'UNRWA avait ouvert l'école "Najda", qui enseigne le français à Beddawi. En 1958 nous avons quitté le Mina pour venir à Beddawi 8 ».

L'atelier de monsieur Burhân se situe dans son ancienne maison, au cœur du camp. Monsieur Burhân n'a jamais commercialisé ses tableaux, qu'il offre à ses amis et parents. L'artiste a déjà partagé plusieurs expositions collectives dans la région, aussi bien qu'en Belgique et en France.

Puis, il y a les autres. Plus jeunes.

Nizar, 32 ans, est né à Beddawi, où il a étudié dans les écoles de l'UNRWA. C'est aussi dans le camp qu'il a initié son éducation artistique, auprès de monsieur Burhân. Il a poursuivi ses études à l'Institut technique de Tripoli, où il a réussi son baccalauréat de design d'intérieur. Ensuite, il a suivi une formation académique à l'université libanaise, à Tripoli, où il a obtenu son diplôme de Beaux-Arts (bac +4). Nizar a financé ses études universitaires en peignant des bâtiments dans le camp. Aujourd'hui, l'artiste est assistant d'enseignement de l'art dans les écoles de l'UNRWA dans les camps de Beddawi et de Nah AlBared. Nizar a déjà participé à plusieurs expositions collectives dans la région. Il a également partagé les expositions en Belgique et en France avec monsieur

8. Entretien réalisé en français (signalons qu'au moment de cet entretien, cela faisait treize ans que monsieur Buhrân n'avait pas parlé français). 
Burhân. Comme ce dernier, Nizar n'a jamais commercialisé ses peintures. Selon lui, «les Palestiniens n'ont pas assez d'argent pour acheter des tableaux » :

« J'essaie toujours de rendre mon travail plus humain. Je n'aime pas peindre la spécificité de la cause palestinienne. Parce que comme ça je peux parler avec les Anglais, les Hollandais, les Japonais, les gens de n'importe où. Je peux parler avec ces personnes quand je fais mon travail en tant qu'individu. Je pense que c'est la meilleure façon d'établir mon langage et d'expliquer ma cause, ma cause palestinienne.

Tout le temps je dessine et je peins à propos de ma cause. Et quand je te parle de ma cause, je ne parle pas seulement de la cause nationale, Intifadas et bombes et tout ça, non, pas seulement ça. Comme un être humain. [...] Mes peintures, chaque chose que je peins est, en général, politique, mais je ne parle pas des événements politiques directement. J'essaie toujours de les rendre plus individuels, quelquefois personnels. Ces peintures sont toutes originaires d'une expérience personnelle. Je dessine mon expérience en tant que Palestinien, mais j'essaie de lui donner un langage humain, qui puisse être lu par les autres, par de plus en plus de personnes. Je ne me pose pas la question de savoir si je vais faire un travail sur la cause palestinienne ou non avant de peindre. Tout simplement je n'ai pas le temps de penser à d'autres choses. Je pense que l'artiste peint toujours quelque chose qu'il a vécu ou senti, tu vois. Je ne peux pas parler d'une chose dont je ne sais pas comment la penser. Comment est-ce que je peux peindre une cause dont je ne sais rien ? Il faut que je vive l'expérience ellemême pour pouvoir l'expliquer, pour produire une expression à son propos. [...]

Quelques travaux parlent de la cause palestinienne, de la cause nationale, de la terre natale. Et quelques autres parlent des expériences personnelles, d'amour. Deux ou trois de mes travaux parlent d'une relation qui était une expérience malheureuse, dont le résultat fut catastrophique. Pourquoi ? A cause de ma nationalité. C'est cela le sujet de mes projets, j'en parle en tant que Palestinien. Je parle des expériences qui étaient mauvaises à cause de ma nationalité, et cela appartient à ma cause. Pas seulement des armes, des bombes. Et c'est de cela que je suis toujours en train de parler dans mon travail ${ }^{9}$ ». 
Iman, 36 ans, est mariée à un Palestinien et habite à Beddawi depuis 10 ans. Ils ont deux enfants, âgés de 9 et 5 ans. Iman fut un des professeurs de Nizar à l'Institut technique de Tripoli. Comme lui, elle a suivi la formation de Beaux-Arts de l'université libanaise. Actuellement, l'artiste est enseignante au Collège national $\mathrm{Al}$-Minieh, à Tripoli. Iman a déjà exposé son travail à Tripoli et à Beyrouth. Occasionnellement, elle vend ses tableaux, mais c'est dans l'enseignement qu'Iman trouve sa principale source financière.

«Quand j'ai épousé un Palestinien et suis venue vivre ici dans le camp, au fil du temps je me suis aperçue que les femmes que je peins ne sont plus les mêmes. C'est une nouvelle femme car indirectement je suis toujours en train de réfléchir aux femmes palestiniennes et à d'autres aspects du peuple palestinien. Comme maintenant je pense toujours au cas palestinien, sans que je m'en rende compte mes chevaux sont devenus des chevaux de guerre, mes femmes des femmes dans la guerre... Le cheval est devenu le cheval de la révolution. Avant, mes chevaux étaient stables, maintenant ils sont toujours en mouvement, en train de sauter, de courir... ${ }^{10}$ ».

Yosof a 39 ans, il est né dans l'ancien camp de réfugiés de Tal el Za'atar. Lui et sa famille ont dû se déplacer à plusieurs reprises pendant la guerre du Liban. En 1982, lors de l'invasion israélienne, sa famille est allée vivre à Balbeque, dans la vallée de la Beqaa. C’est là que Yosof a rencontré deux artistes iraquiens qui lui ont appris le dessin et la peinture. En 1984, il s'est installé dans le camp de Beddawi. Yosof a commencé des études d'art dans un institut à Tripoli, mais il n’a pas achevé ses études. Selon lui, «les temps étaient durs». Plus tard, Yosof est parti à Damas, où il a suivi une formation d'un an et demi à l'Institut des Beaux-Arts. L'artiste a déjà exposé son travail dans les deux camps du Nord, à Tripoli et à Beyrouth. Il est le seul artiste Palestinien de Beddawi à commercialiser ses tableaux. Il fait aussi des illustrations de livres pédagogiques :

"Quand je considère mon travail comme une carrière, il n'y a pas de problème, je peux peindre et parler de quelque chose d'autre. Mais quand j'ai envie de peindre mon expérience, mes idées, mes sentiments, je ne peux parler que de la cause palestinienne. Parce que je suis né Palestinien. Quelquefois, quand j’ai besoin de faire un peu d'argent, je fais des paysages, etc. Mais quand je veux parler de ce à quoi je crois, par exemple une idée, mon opinion, des choses qu'il y a dans ma tête... ».

Samir, 33 ans, est né dans le camp de Beddawi. C'est un ami proche de Nizar. En 1982, pendant la guerre civile, sa famille est partie en Syrie. A

10. Cet entretien, ainsi que les deux suivants, ont été réalisés en arabe. Nizar les a traduits simultanément en anglais. Notre traduction vers le français. 
Damas, il a fréquenté une école de l'Organisation de libération de la Palestine (OLP) destinée aux enfants des martyrs palestiniens, où il a commencé à apprendre le dessin. A la fin de ses études élémentaires, Samir est retourné au Liban avec sa famille. Il a commencé une formation d'architecture mais n'a pas pu la mener à terme : son père et son frère aîné étant morts pendant la guerre, c'était à lui de subvenir aux besoins financiers de sa famille. Samir a épousé sa cousine, qui habite dans le camp avec lui. Ils ont deux filles, de 5 et 2 ans. Il est propriétaire d'un petit commerce à Tripoli, et a pour bobby la peinture et la pyrogravure sur bois.

«Je ne peux pas ne pas parler de la Palestine dans mes peintures. Je ne peux parler que de notre situation [...]. Je veux que les gens sachent que notre peuple aime la vie et qu'il fait des grands efforts pour continuer vivant, et qu'il essaie de vivre en paix. On essaie toujours de rendre la vie meilleure, mais à chaque fois qu'on essaie on trouve un nouvel obstacle. On essaie encore et encore et il y a encore une nouvelle guerre. [...] Les gens peuvent aller sur la lune mais ils ne peuvent rien faire à propos de notre terre natale. Jusqu'à présent les sionistes essaient à tout pris de nous tuer. C'est bien qu'à ton âge tu puisses raconter notre situation à l'Occident ».

\section{Précisions sur l'enquête}

C'est d'abord dans le cadre de la préparation de mon mémoire de DEA et, ensuite, de l'élaboration de ma thèse de doctorat, que j'ai réalisé trois travaux de terrain dans le camp de Beddawi, au Nord-Liban. A l'occasion de mon premier séjour, en avril 2004, j'ai vécu pendant un mois au sein d'une famille palestinienne du camp. Au cours du deuxième séjour, j’ai résidé à Beyrouth, où j'ai suivi un stage de huit mois au siège de l'UNRWA. Chaque dimanche, pourtant, je suis retournée déjeuner chez ma famille d'accueil, par amitié. Finalement, en avril 2007, je suis de nouveau retournée chez eux, cette fois-ci pour une période de trois semaines.

Dès le départ, j’ai été chaleureusement accueillie par la famille de M. Abd et Soraia. Le fait d'être une jeune femme, voyageant seule, ayant le même âge qu'une des filles de la maison, a sûrement facilité mon acceptation dans ce foyer. Les affinités réelles qui existent entre nous ont aussi joué un rôle important dans ma relation avec la famille, qui a finit par m'adopter. Je ne peux pas m'abstenir d'utiliser le terme « adopter », peut-être perçu par les chercheurs plus conventionnels comme excessif. De la nourriture que Soraia préparait pour moi à chaque fois que je quittais le camp pour Beyrouth, aux ressemblances physiques qu'elle revendique entre moi, elle-même et ses deux filles, en passant par les appels téléphoniques réguliers de la famille quand je ne suis pas au Liban, le tout couronné par l'argent que Soraia et M. Abd ont tenu à 
m'offrir lors de mon dernier départ, en me disant : «Si tu ne l'acceptes pas c'est parce que tu ne te considères pas comme notre fille »... toutes ces petites choses m'empêchent de m'exprimer autrement. Si je le mentionne ici c'est parce que, en tant que chercheuse de terrain, il me semble important d'expliciter la place qui m’a été assignée et que j'ai naturellement assumée.

Le texte qui va suivre est un extrait de mon journal de terrain. Il a été écrit à l'occasion de mon dernier séjour chez la famille de M. Abd et Soraia, en avril 2007. Je leur avais exprimé mon envie de décrire une journée typique de leur quotidien. Non seulement ils ont approuvé cette idée mais, enthousiastes, ils m'ont aidé à me souvenir des détails de leur vie quotidienne. Cela leur a toujours fait plaisir de savoir que je tenais à montrer le côté humain de leur réalité. Malheureusement, ce quotidien a été brusquement bousculé par les affrontements qui ont opposé l'armée libanaise aux militants du groupe Fatah Al-Islam dans le camp palestinien voisin de Nahr Al-Bared, quelques semaines plus tard.

Les combats ont commencé le 20 mai et se sont prolongés jusqu'au 2 septembre 2007. En raison de ces combats, la quasi-totalité des 35000 réfugiés de Nahr Al-Bared a fui le camp par vagues successives. Nah Al-Bared est en quelque sorte le grand frère de Beddawi. En raison de leur proximité géographique, il y a entre les réfugiés des deux camps des liens familiaux, d'amitié et de travail. Ainsi, il était naturel que les personnes déplacées de Nahr Al-Bared trouvent refuge à Beddawi. Selon les chiffres officiels de l'UNRWA, au 7 août 2007 Beddawi accueillait 13775 réfugiés issus du camp voisin. En d'autres termes, le nombre de réfugiés dans le camp de Beddawi a pratiquement doublé pendant ces derniers mois. Cela représente une lourde charge pour le camp, déjà surpeuplé 11 .

Je ne peux pas m'empêcher de penser à la pertinence du titre du livre de Rosemary Sayigh, Too many enemies ${ }^{12}$. En raison de la vulnérabilité de leur condition, une fois de plus mise à jour par les derniers événements, Soraia et M. Abd songent à quitter le pays. Soraia, bien qu'étant l'une des survivantes du massacre du camp palestinien de Tel Zaatar en 1976, n'avait jamais manifesté l'envie de partir du Liban, qu'elle décrivait comme "un beau pays quand il n'y a pas de bombes». M. Abd disait qu'il ne quitterait le Liban que pour rentrer en Palestine. Désormais, les projets de la famille ont changé : ils comptent s'installer à Abou Dhabi (Emirats arabes unis) au plus tôt. Cela pourrait être possible grâce aux réseaux familiaux de Soraia, à la différence de la majorité des familles du camp de réfugiés, pour qui l'émigration n'est pas une solution facile.

11. Il y avait à la même date 26182 personnes déplacées dans le nord dont la majorité dans le camp et aux proches alentours; en réalité, la population du « camp » a donc plus que doublée.

12. Rosemary Sayigh, Too Many Enemies: The Palestinian Experience in Lebanon, Londres, Zed Books, 1994. 


\section{Une journée à Beddawi. Carnet de route}

Vers quatre heures du matin, alors que les maisons du camp sont encore silencieuses, les mosquées de Beddawi appellent leurs fidèles pour la première prière de la journée. Les six ou sept mosquées du camp résonnent toutes en même temps, avec un petit décalage de quelques minutes entre elles. Le résultat est un concert non affiné, qui vient réveiller ceux qui désirent prier et perturber le sommeil de ceux qui ne sont pas encore habitués aux sons de Beddawi.

A l'extérieur du camp, en ville, les mosquées doivent s'en tenir à une prière ordinaire. Ici, au contraire, elles peuvent faire entendre des chansons qui rappellent la vie du Prophète, ou encore des versets du Coran, avant et après le «Allah u Akbar». Pendant cette demi-heure musicale, les fidèles de Beddawi ont le temps de se préparer pour l'oraison. Chaque matin, de ma chambre, j'entends M. Abd se lever, faire ses ablutions, s'habiller, boire un verre d'eau et partir en direction de la mosquée la plus proche.

Tout est encore sombre. Les rues et les ruelles de Beddawi sont tranquilles. Seuls quelques passants, partis en direction des mosquées, viennent troubler un calme complet. Rien ne nous indique que, d'ici quelques heures, bruits et mouvements empliront l'espace du camp.

Vers cinq heures du matin, M. Abd est de retour chez lui. Sa femme, Soraia et sa fille Rola, sont encore endormies. Il profite de ce moment de quiétude pour se consacrer à la lecture. Deux sujets l'intéressent : les textes sacrés et les livres de géographie. Professeur des écoles de l'UNRWA, M. Abd est diplômé d'un master en géographie. Il songe à faire un doctorat quand il aura sa retraite, d'ici six mois.

Une heure plus tard, le téléphone sonne. Il s'agit de l'une des amies de Soraia, qui l'appelle tous les jours, à la même heure, pour la réveiller. Levée, Soraia prépare le café turc. L'arôme du café mélangé à la cardamome emplit la maison. A six heures et demie du matin, Anissa, une voisine de l'immeuble d'à côté, arrive. Assises par terre, les deux amies fument des cigarettes et se racontent ce que la journée leur réserve.

Anissa et Soraia habitent d'anciens immeubles bâtis par l'OLP. Les deux immeubles sont identiques. Leurs façades sont jaunes et dissimulent deux appartements par étage. Chaque logement est pourvu d'un petit balcon. Debout sur le balcon, au printemps, on aperçoit un champ de petites fleurs jaunes, entouré par des montagnes enneigées. La beauté de cet horizon contraste avec la saleté des rues de Beddawi. Alors que les maisons de Soraia et de ses voisines sont très propres et bien arrangées, l'espace commun de l'immeuble est extrêmement sale. Sur les escaliers : des papiers de bonbons et de chips, des moutons de poussière, des paquets de cigarette vides... A la sortie 
de l'immeuble, il n'est pas rare de croiser le chemin d'un rat (jardon), ou de trouver le corps d'un autre moins chanceux, tué par un passant et abandonné sur place. Soraia rit de la façon dont je cours quand je sors de l'immeuble, par peur de rencontrer un jardon qui se cache dans la cage d'escalier.

Parfois, Mayada, la voisine de l'étage au dessus, se joint à Soraia et Anissa. Elle arrive vers sept heures. Libanaise chrétienne, Mayada a épousé un Palestinien de Beddawi. Ils ont deux enfants. C'est dans le foyer de Soraia que Mayada a trouvé son confort durant les quatre ans que son mari a passé en Angleterre pour gagner de l'argent. Comme il dit, il n'y est pas allé en avion, mais « de l'autre façon ». Complètement intégrée dans le camp, Mayada partage le quotidien de ses voisines palestiniennes. D'habitude, elle fume et papote avec Soraia jusqu'à huit heures du matin. Puis, elle monte les escaliers pour rentrer vaquer à ses tâches ménagères. A cette heure-ci, Anissa est déjà partie travailler depuis longtemps.

Revenons à M. Abd. A sept heures du matin, il quitte sa chambre et traverse le salon enfumé pour se rendre à son travail. Sur le mur extérieur de son immeuble, quelqu'un a écrit Germany. Probablement son fils Basel, qui habite en Allemagne et qui est venu leur rendre visite l'été passé. Sur le mur de l'immeuble d'en face, le nom « Palestine » est marqué en caractères arabes, avec sa traduction en anglais au-dessous. Très simples, ces graffitis ont été faits avec un marqueur noir. A côté de ce mur, la pancarte indique une petite fabrique de chocolat, nommée « Glamour».

La maison de M. Abd et Soraia se situe sur une rue large, en comparaison avec les passages de l'intérieur du camp. Cette rue est perpendiculaire à la grande rue principale, qui délimite le camp de Beddawi. Ainsi, à strictement parler, leur maison n'est pas dans le territoire du camp de réfugiés. Mais au quotidien, cette frontière entre le dedans et le dehors disparaît. Il s'agit d'un espace liminaire, qui ne se situe ni dans l'espace propre du camp, ni dans les rues claires de l'extérieur.

Plus loin en descendant, nous arrivons sur la rue principale du camp de Beddawi. La lumière du soleil est encore douce. Petit à petit, le calme de ce matin laisse place au mouvement des écoliers et des travailleurs. C'est l'heure des minibus remplis d'élèves et de parents qui tiennent leurs enfants par la main sur la route de l'école. Les transports de l'UNRWA partagent cet espace avec celui des écoliers. Ils viennent chercher les fonctionnaires pour les emmener au camp voisin de Nah Al-Bared, à Tripoli, ou au bureau principal de l'Agence à Beyrouth.

A cette heure-ci, les taxis collectifs, appelés « services ", commencent à chercher des clients. Les services sont le système de transport collectif le plus répandu au Liban. Ce sont des vieilles Mercedes, qui klaxonnent à chaque fois 
qu'elles passent devant un passager potentiel - ce qui crée une ambiance sonore très particulière. Le prix d'un service est de 1000 livres libanaises par personne (moins d'un dollar), et chaque voiture peut emmener jusqu'à cinq, voire six passagers en même temps. A Beddawi, ces taxis assurent l'allerretour jusqu'à Tripoli. Au centre de cette ville, il y a même le «parking de Beddawi », rendez-vous de ceux qui s'en retournent au camp. Par solidarité, mais aussi pour des raisons de sécurité, les Palestiniens de Beddawi refusent les taxis qui ne sont pas conduits par un chauffeur de leur camp.

M. Abd travaille comme responsable des bibliothèques des écoles de l'UNRWA des camps de Beddawi et Nahr Al-Bared. Les jours où il travaille à Beddawi, il continue son chemin à pied dans la rue principale. Il longe les murs d'une école de l'Agence pour y entrer dans une ruelle perpendiculaire à gauche. Les murs de cette école sont investis par diverses fresques murales. Il s'agit d'une initiative récente des artistes plasticiens de Beddawi. Ils l'ont demandé à l'école et négocié avec un qashaf 13 du camp le matériel nécessaire à ces peintures. Cet effort collectif a donné un ensemble coloré d'une dizaine de fresques murales. Chaque artiste a conçu sa propre fresque avec pour thème commun la Palestine : un verset du Coran appelant à la résistance, un olivier encadré par les couleurs du drapeau palestinien, deux chevaux dans la brume, un visage entouré par le keffieb ${ }^{14}$ palestinien... Autant de façons de manifester que la Palestine est toujours vivante dans l'esprit de ce camp. Une phrase en arabe inscrite au-dessus des fresques unifie l'ensemble : « La Palestine libre de la mer à la rivière ».

Les jours où M. Abd travaille à Nahr Al-Bared, il s'y rend dans le bus de l'UNRWA avec d'autres professeurs et fonctionnaires de l'Agence. Au croisement de sa rue avec la rue principale, en face de la station d'essence du camp de Beddawi, il attend le bus qui l'amènera au camp voisin. A l'extrémité gauche de la station, nous avons une miniature en béton de la mosquée $A l-A q s a$. Située à Jérusalem, $A l$-Aqsa est, avec Qobat al-Sakhrah, l'une des mosquées les plus importantes pour les musulmans, notamment pour les Palestiniens. Son dôme large et doré se reconnaît facilement aux nombreuses fresques murales du camp. Al-Aqsa est un symbole de l'islam, mais aussi de la Palestine. A côté de la petite mosquée, il y a un monument composé de ce qu'il reste d'une bombe israélienne tombée sur le camp en 1982. Cette bombe a coûté le bras de l'un des artistes plasticiens de Beddawi, Samir. Etonnée par le handicap de son père, la petite Soubkheia, âgée de quatre ans, lui promet qu'elle ira en Israël chercher le bras qui lui manque. A l'autre extrémité de la station d'essence, il y a quelques pneus empilés. Ces pneus sont utilisés pour tenir les quelques toits de zinc qui persistent dans le camp.

13. Sorte de scouts.

14. Coiffe traditionnelle des paysans et des Bédouins d'Arabie et du Moyen-Orient, devenue l'emblème des Palestiniens. 
A huit heures et demi du matin, Rana, une amie de Soraia, vient la chercher en voiture. Soigneusement habillées et maquillées, les deux amies partent vendre des cosmétiques au porte-à-porte, dans les environs de Beddawi et à Tripoli. Rana est une très belle jeune femme de 24 ans. Il y a quelques années, elle a épousé son cousin germain. Ils ont eu deux filles, toutes les deux avec de graves handicaps physiques et mentaux. Consciente du rôle de la consanguinité dans le handicap de ses filles, Rana refuse d'avoir d'autres enfants. C'est la raison pour laquelle son mari a décidé de la quitter. Comme le veut la tradition, c'est à elle d'abandonner maison et enfants et de rentrer chez ses parents.

A 11 heures du matin, Rola, la fille de Soraia, et sa copine Houda se réveillent. Houda est aussi une voisine de l'immeuble d'à côté. Elle a 20 ans et vient tous les jours dormir chez Soraia. Les trois femmes dorment ensemble dans la même chambre, sur des matelas posés par terre. La maison de Soraia a trois chambres, dont deux ont été réaménagées récemment. L'une est la chambre où M. Abd dort. Elle compte deux lits, le sien et un autre, qui est libre. L'autre chambre comporte également deux lits. Elle a été décorée avec des rideaux roses et les draps qui vont avec. C'est ma chambre. Soraia dit qu'un lit est à moi, et l'autre est à sa fille Lina, qui a mon âge. Après avoir fini ses études à Sibline l'année passée, Lina est partie travailler à Abou Dhabi. La troisième chambre n'est pas meublée. Soraia explique qu'elle préfère dormir par terre, peut-être par habitude (elle continue également à préparer la nourriture et à manger par terre, ignorant les canapés et les tables de la maison).

Au réveil, quand il n'y a plus personne à la maison, Rola et Houda mettent de la musique arabe très forte et s'occupent des tâches ménagères. Rola a raté son bac l'année passé. Elle est catégorique quand elle dit qu'elle n'aime pas étudier. Mais sa mère l'oblige à fréquenter des cours privés, pour qu'elle puisse le tenter à nouveau cette année. Les parents de Houda sont moins concernés par son éducation. Elle a quitté l'école il y a longtemps. Elle dit qu'elle non plus n'aime pas les études. La plupart des amies de Rola ont quitté l'école avant d'arriver jusqu'au bac. Peut-être qu'elles ne voient pas de réel intérêt à étudier...

A 12h30, Houda descend les escaliers et traverse la ruelle qui sépare l'immeuble de Soraia du sien. Il s'agit d'une rue très courte, mais assez large. Très peu de voitures passent par cette rue, transformée en une sorte de terrain de jeux pour les enfants du voisinage. A l'une de ses extremités, il y a un arc en béton. Il offre un joli cadre aux enfants qui jouent à cache-cache, au football et à la marelle. C'est un lieu de joie dans le camp. Une fois rentrée chez elle, Houda s'occupe du ménage avec sa mère.

Une heure plus tard, Rola descend la rue principale, où elle prend un « service » pour aller chez son professeur. Le soleil frappe fort et la rue principale est mouvementée. La plupart des femmes dans la rue sont voilées, mais 
quelquefois on croise une femme avec les cheveux au vent. Il y a trois ans, Rola a décidé de mettre le voile. La nouvelle avait étonné ses proches, puisqu'elle n'a jamais été particulièrement religieuse. De plus, sa mère et sa grande soeur ne sont pas voilées. La jeune fille n'a pas donné de raisons à cette décision si soudaine. Quand on lui posait la question, elle disait simplement : «c'est comme ça». Deux ans plus tard, après avoir séjourné quelques mois à Abou Dhabi, Rola a enlevé son voile. Cette fois-ci, elle en dit plus, qu'elle aimait faire du jet ski avec ses nouveaux amis, qu'elle rêve de quitter le camp de réfugiés pour le cosmopolitisme d'Abou Dhabi. Elle dit qu'ici les gens parlent beaucoup et que tout est interdit.

A 14h30, Soraia est de retour à la maison. Une fois rentrée du travail, elle prépare la nourriture du lendemain. Assise par terre sur une couverture dans le salon, elle prépare les plats traditionnels avec une patience exemplaire. Elle arrache les petites feuilles de thym une par une, pour faire une conserve avec du citron. D'autres fois elle roule un mélange de viande et de riz dans des feuilles de vigne. Il n'est pas rare qu'elle épluche des petits pois et coupe les carottes en petits morceaux. Quand M. Abd rentre du travail, une demi-heure plus tard, le repas préparé la veille est déjà chauffé. Il mange et fait sa sieste.

Deux fois par semaine, M. Abd rentre de l'école un peu plus tôt, à $13 \mathrm{~h} 30$. Ces jours-là, il échange son costume contre un survêtement de sport, cadeau que son fils lui a ramené d'Allemagne. M. Abd part à la montagne avec un groupe d'amis de Beddawi. Ils sont plus jeunes que lui, il a déjà la soixantaine. La montagne n'est pas loin du camp et le groupe s'y rend à pied. Au printemps, la route est remplie de coquelicots. M. Abd en profite pour ramasser de la sauge, une herbe qui, nous dit-il, est très bonne pour la mémoire. Il la boit, mélangée avec du thé noir. M. Abd ramasse également de la menthe sauvage et d'autres herbes aromatiques. Soraia se plaint de la quantité d'herbes qu'elle accumule dans sa cuisine, mais il explique que c'est pour offrir aux personnes qui n'ont pas l'occasion d'aller les chercher dans la montagne. Sa promenade dans la nature dure environs trois heures.

Vers 15h, Anissa repasse chez Soraia pour fumer une autre cigarette avec sa voisine. Il s'agit d'une visite courte, qui ne dure guère plus de 10 minutes. Certains jours, vers $20 \mathrm{~h}$, c'est au tour de Soraia de lui rendre visite. Comme d'habitude, elles fument et discutent. Vers 16h, Rola rentre de son cours privé. Elle consacre le reste de l'après-midi à regarder la télévision et à discuter au téléphone avec ses amies. Quelques fois, vers $20 \mathrm{~h}$, elle se rend chez Houda où il y a un ordinateur connecté à Internet. Alors Rola en profite pour regarder ses emails et chatter sur msn avec ses amis d'Abou Dhabi. Beddawi compte six ou sept webcafés qui ne sont fréquentés que par les jeunes garçons du camp. Deux ans auparavant, il y avait un webcafé très familial, où j'allais souvent avec Rola. Maintenant qu'il est fermé, les jeunes filles convenables de Beddawi sont obligées de trouver un autre moyen de naviguer sur Internet. 